\title{
Analysis of Algebraic Expressions Derived from Genetic Multivariate Polynomials and Support Vector Machines: A Case Study
}

\author{
Ángel Kuri-Morales ${ }^{1}$ and Iván Mejía-Guevara² \\ ${ }^{1}$ Departamento de Computación, Instituto Tecnológico Autónomo de México, \\ Río Hondo No. 1 , \\ 01000 D. F., México \\ akuri@itam.mx \\ ${ }^{2}$ Posgrado en Ciencia e Ingeniería de la Computación, Universidad Nacional Autónoma de \\ México, IIMAS, Ciudad Universitaria, \\ 04510 D. F., México \\ imejia@uxmcc2.iimas.unam.mx
}

\begin{abstract}
We discuss how algebraic explicit expressions modeling a complex phenomenon via an adequate set of data can be derived from the application of Genetic Multivariate Polynomials (GMPs), on the one hand, and Support Vector Machines (SVMs) on the other. A polynomial expression is derived in GMPs in a natural way, whereas in SVMs a polynomial kernel is employed to derive a similar one. In any particular problem an evolutionary determined sample of monomials is required in GMP expressions while, on the other hand, there is a large number of monomials implicit in the SVM approach. We make some experiments to compare the modeling characterization and accuracy obtained from the application of both methods.
\end{abstract}

\section{Introduction}

Function approximation from experimental data is an issue that many researchers have tackled in the past. The specification of certain functions given a dataset is commonly used to model interesting phenomena. Genetic Multivariate Polynomials (GMPs) modeling [2] relies on specific polynomial functions given a dataset. A simple and explicit algebraic expression is derived form the application of GMPs. The relationship and specification of the explanatory variables arises in a natural way. In classical methodologies a full polynomial model is attained from an exhaustive exploration of the linear combinations of the set of monomials corresponding to the independent variables. In this case the number of possible combinations can increase unmanageably. In the latter case, it turns out to be not only impractical but impossible to tackle the modeling problem for even a modest number of independent variables. This drawback is eliminated in GMPs by a) Changing the commonly used approximation least square error norm by a minimax norm and b) Employing Genetic Algorithms (GAs) to reduce the number of monomials in the final model. This is achieved 
without losing the capability of this model ("learning machine") to represent the phenomenon under analysis.

Another approach that has been proved to be successful relies on Support Vector Machines (SVMs). This methodology relies on finding a functional that is nonlinear in the input space, but linear in a higher dimensional feature space and is capable of making classification, regression or function approximation [10]. Acting, in effect, as a Neural Network with one hidden nonlinear layer, SVMs, as all other connectionist schemes, have been labeled "black boxes" [1], since the complex relationships between explanatory variables are not explicit in its structure. However, one of the main goals of this paper is to show that we are able to build algebraic explicit expressions (which depend on the appropriate selection of the kernel function) from such SVMs. In particular, we use a polynomial kernel that allows us to find an expression comparable to the ones arising from GMPs.

In section 2, we point out the main theoretical characteristics of GMPs and the advantage of using genetic algorithms (GAs). In section 3, the general foundations of SVMs are presented. Particularly, we discuss the way in which, via SVMs, one may find a solution to the nonlinear regression problem. We also show how a polynomial kernel can be used in SVMs in order to derive an explicit algebraic expression from the support vector solution. In section 4, some experiments and results are shown applying both methodologies to a set of representative regression problems. Finally, in section 5 we advance some conclusions and discuss the results of the previous section.

\section{Genetic Multivariate Polynomials}

Given a training sample $\varsigma=\left\{\left(\mathbf{x}_{i}, y_{i}\right)\right\}_{i=1}^{N}$, where $\boldsymbol{x}_{i}$ denotes the $i$-th input vector pattern and $y_{i}$ its corresponding target output, the goal of GMPs is to find an explicit algebraic expression of the form:

$$
F\left(v_{1}, \ldots, v_{n}\right)=\sum_{i_{1}=0}^{d_{1}} \ldots \sum_{i_{n}=0}^{d n} c_{i_{1}} \cdots i_{n} v_{1}^{i_{1}} \ldots . v_{n}^{i_{n}}
$$

The sets of coefficients in (1) (denoted as $\mathbf{C}^{\prime}$ ) yield an explicit mathematical relationship between the independent variables $\left(\boldsymbol{x}_{i}\right)$ and dependent variables $\left(y_{i}\right)$. This approximation problem has been the subject of many studies in the past and it has been pretty much solved except for certain practical issues. First of all, we have to contend with the fact that the cardinality of $\mathbf{C}^{\prime}$ (which we denote as $\gamma^{\prime}$ ) is large enough to make it unwieldy for all except the simplest cases. Second, considering a certain floating point number representation ( 8 bytes for example), the storage of a hypothetical solution will normally require a huge number of bytes. Third, and most important, the usual numerical methods with which these approximations are normally tackled imply the solution of systems of linear equations of large order with the consequent inevitable numerical instability. Finally, fitting a relatively large set of data under least squares error measure leads to Hilbert matrices which are known to be particularly sensitive to rounding error [13]. Hence, even though it is theoretically 
possible to solve the approximation problem even for large samples, in practice it is both impossible and impractical to do so with the usual methods [2]. The approximation given by GMPs replaces the equation (1) by another one in which we retain the algebraic polynomial of the solution while removing the need for large $\gamma$ ' by introducing the constant $\boldsymbol{\mu}$ as shown by:

$$
F\left(v_{1}, \ldots, v_{n}\right)=\sum_{i_{1}=0}^{d_{1}} \ldots \sum_{i_{n}=0}^{d_{n}} \mu_{i_{1} \ldots i_{n}} c_{i_{1} \ldots i_{n}} v_{1}^{i_{1}} \ldots . v_{n}^{i_{n}}
$$

The coefficients are represented as $c_{i_{1} \ldots i_{n}}$ where $d_{i}$ represents the maximum allowed exponent of the $i$-th independent variable. The elements of $\boldsymbol{\mu}$ determine whether a given monomial in (2) is to be included in the purported polynomial solution. For this reason we must establish a priori the cardinality of $\boldsymbol{\mu}$. Obviously, we want to have a manageable number of coefficients so that we do not longer need to contend with the problems derived from large storage needs. Therefore, we will replace the least squares (or $\mathrm{L}_{2}$ ) norm with less common minimax (or $\mathrm{L}_{\infty}$ ) norm. In so doing we will avoid the need to solve large systems of equations and get around the inherent precision problems. The minimax norm is not as popular as least squares because the approximation algorithms are normally slower. In what follows we give a brief account of the principles behind the minimax multivariate approximation algorithm. Once this is done we will discuss the genetic algorithm that allows us to determine $\mathbf{C}^{\prime}$ for a given $\gamma$.

\subsection{The Ascent Algorithm}

The algorithm to approximate the sample under the minimax norm is based on three observations: a) The minimax approximation coefficients of a set $M$ of size $m$ are uniquely determined by finding the adequate signs of the errors for the elements of the set, b) if a larger set $N$ of size $n$ has elements outside the minimax solution it is always possible to exchange one of elements in $M$ by an element in $N$ such that the minimax condition is still met for the new $M$ and c) Continuing exchanges will eventually lead to a target set $M$ which satisfies the minimax norm for all elements in $N$.

In what follows we denote with $F(\boldsymbol{x})$ the function which is able to minimize the error $\ell_{\varphi}=\max \left|F_{i}\left(x_{i}\right)-d_{i}\right|$. In order to find the approximator of (2) we map the vectors $\boldsymbol{x}$ to a higher dimensional space yielding matrix $\mathbf{V}$ of dimension $p \times N$ where $p=\prod_{i=1}^{n}\left(1+d_{i}\right)$. Let us arbitrarily select a sub-matrix of $\mathbf{V}$ of size $\mathrm{m} \times \mathrm{m}$ (call it $\mathbf{V}^{\prime}$ ). Then we solve the following system:

$$
\left[\begin{array}{cccc}
\eta_{1} & \left(v_{1}^{0} \ldots v_{n}^{0}\right)_{1} & \ldots & \left(v_{1}^{d_{1}} \ldots v_{n}^{d_{n}}\right)_{1} \\
\eta_{2} & \left(v_{1}^{0} \ldots v_{n}^{0}\right)_{2} & & \left(v_{1}^{d_{1}} \ldots v_{n}^{d_{n}}\right)_{2} \\
\ldots & \ldots & \ldots & \ldots \\
\eta_{m} & \left(v_{1}^{0} \ldots v_{n}^{0}\right)_{m} & & \left(v_{1}^{d_{1}} \ldots v_{n}^{d_{n}}\right)_{m}
\end{array}\right]\left[\begin{array}{c}
\ell_{\theta} \\
c_{1} \\
\ldots \\
c_{m-1}
\end{array}\right]=\left[\begin{array}{c}
y_{1} \\
y_{2} \\
\ldots \\
y_{m}
\end{array}\right]
$$


Denoting the approximation error for the $i$-th vector as $\ell_{i}$ and the largest absolute such error as $\ell_{\theta}$ we may define $\ell_{i}=\eta_{i} \ell_{\theta}$; clearly, $\ell_{i} \eta_{i} \leq \ell_{\theta}$. We also denote the elements of row $i$ column $j$ of (3) as $\boldsymbol{\delta}_{i j}$ and the $i$-th cofactor of the first column as $\boldsymbol{\kappa}_{i}$. From Cramer's rule we immediately have:

$$
\ell_{\theta}=\frac{\left|\begin{array}{ccc}
y_{1} & \ldots & \delta_{1 m} \\
\ldots & \ldots & \ldots \\
y_{m} & \ldots & \delta_{m m}
\end{array}\right|}{\eta_{1} \kappa_{1}+\ldots+\eta_{m} \kappa_{m}}
$$

To minimize $\ell_{\theta}$ we have to maximize the denominator of (4). This is easily achieved by a) selecting the maximum value of the $\boldsymbol{\eta}_{i}$ 's and b) making the signs of the $\boldsymbol{\eta}_{i}$ 's all equal to the signs of $\boldsymbol{\kappa}_{i}$ 's. Obviously the $\boldsymbol{\eta}_{i}$ 's are maximized iff $a b s\left(\boldsymbol{\eta}_{i}\right)=1$ for $i=1, \ldots, m$ which translates into the well known fact that the minimax fit corresponds to approximation errors of the same absolute size. On the other hand, to achieve (b) we must simply set the signs of the $\boldsymbol{\eta}_{i}$ 's to those of the cofactors. Making $\sigma_{i}=\operatorname{sign}\left(\kappa_{i}\right)$, system (3) is simply re-written as:

$$
\left[\begin{array}{ccc}
\sigma_{1} & \cdots & \delta_{1 m} \\
\cdots & \cdots & \cdots \\
\sigma_{m} & \cdots & \delta_{m m}
\end{array}\right]\left[\begin{array}{c}
\ell_{\theta} \\
\cdots \\
c_{m-1}
\end{array}\right]=\left[\begin{array}{c}
y_{1} \\
\cdots \\
y_{m}
\end{array}\right]
$$

Once having all the elements in (5) it suffices to solve this system to obtain, both, the value of $\ell_{\theta}$ and the coefficients $\mathbf{C}^{\prime}$ which best fit the elements of $\boldsymbol{x}$ in the minimax sense. To obtain the coefficients for the whole sample we apply the Exchange Algorithm (EA) (for details see [2]).

\subsection{Genetic Algorithm}

Another reason to choose the minimax norm is that the EA is not dependent on the origin of the elements in $\mathbf{V}$. We decided them to be monomials of a full polynomial but it makes no difference to the EA whether the $v_{i}$ are gotten from a set of monomials or they are elements of arbitrary data vectors. This is important because the number of monomials in (3) grows geometrically. One way to avoid the problem of such coefficient explosion is to define $\gamma$ a priori and then properly select which of the $\gamma$ ' possible ones these will be. There are $\left(\begin{array}{l}\gamma^{\prime} \\ \gamma\end{array}\right)$ possible combinations of monomials and even for modest values of $\gamma$ an exhaustive search is out of the question. This optimization problem may be tackled using genetic algorithms as follows.

The genome of the GA is a binary string of size $\gamma^{\prime}$. Every bit in it represents a monomial. If the bit is ' 1 ' it means that the corresponding monomial remains while, if it is ' 0 ', such monomial is not to be considered. This simple strategy corresponds to determining the values of $\mu$ in equation (2). All one has to ensure is that the number of 1 's is equal to $\gamma$. 
It is well known that an elitist GA will converge to a global optimum [3]. It has also been shown that a variation called Vasconcelos' GA (VGA) displays superior behavior on a wide range of functions [4]. VGA uses: a) Deterministic selection, b) annular crossover, c) uniform mutation. All results reported here are based on VGA's application.

\section{Support Vector Machines}

SVMs were developed by Vapnik et al [5] based on statistical learning theory. Particularly, a SVM is an approach to the problem of structural risk minimization, based on the fact that the generalization error rate is bounded by the sum of the training-error rate and a term that depends on the Vapnik-Chervonenkis (VC) dimension [6].

SVMs have been successfully used in practical applications [7]. In the case of classification tasks, the objective is to build a linear surface that separates points belonging to different classes. The objective in regression problems is similar, but in this case the data is enclosed in a $\varepsilon$-tube of the function approximation.

Given the training sample $\varsigma$, the goal of SVMs for non-linear regression problems is to find a function $f(\boldsymbol{x})$ that has at most $\varepsilon$ deviation from the actually obtained targets $\hat{y}_{i}$ for each element of $\varsigma$ and, at the same time, is as flat as possible. In other words, errors are disregarded as long as they are less than $\varepsilon$. This functional has the form $f(\mathbf{x})=\boldsymbol{\omega} \mathbf{x}+b$ with $\mathbf{w} \in \mathfrak{R}^{k}, b \in \mathfrak{R}$. As in the case of classification problems, $f(\boldsymbol{x})$ can be obtained by solving a quadratic optimization problem (QOP) of the form [8]:

$$
\begin{aligned}
& \underset{w, b, \xi}{\operatorname{Min}_{w, \xi}} \frac{1}{2}\|w\|^{2}+C \sum_{i=1}^{N}\left(\xi_{i}+\xi_{i}^{*}\right) \\
& \text { subject to: } \\
& y_{i}-w x-b \leq \varepsilon+\xi_{i} \\
& w x+b-y_{i} \leq \varepsilon+\xi_{i}^{*} \\
& \xi_{i}, \xi_{i}^{*} \quad \geq 0 \\
& \text { for } i=1,2, \ldots, N
\end{aligned}
$$

According to this formulation, the objective is to minimize the empirical risk $R_{e m p}=\frac{1}{N} \sum_{i=1}^{N} L_{\varepsilon}\left(y_{i}-\hat{y}_{i}\right)$, where $L_{\varepsilon}\left(y_{i}-\hat{y}_{i}\right)$ is called the $\varepsilon$-insensitive loss function ${ }^{1}$.

The formulation (6) is known as the primal problem for nonlinearly separable patterns, where the constant $C>0$ determines a trade off between the flatness of $f(\boldsymbol{x})$ and the tolerated amount of points which deviations larger than $\varepsilon$. The appropriate selection of $C$ is a very important issue not only in regression, but in classification problems [9] for an appropriate approximation. The value of $\varepsilon$ is inversely proportional to

\footnotetext{
${ }^{1}$ Other loss functions may be used, but we use the most common $L_{\varepsilon}\left(y_{i}-\hat{y}_{i}\right)=|\xi|-\varepsilon$ if $|\xi|>\varepsilon$ and 0 , otherwise.
} 
the number of support vectors (sv) [10], which are a subset of $\varsigma$, resulting from the solution of (7) and which defines completely $f(x)$; i. e., increasing $\varepsilon$-insensitivity decreases the number of sv. In this paper we use the approach of [11] for the selection of $C$ and $\varepsilon$, where $C=3 \sigma_{d}$ and $\varepsilon=\psi \sigma \sqrt{\ln (N) / N} ; \sigma_{d}, \sigma$ and $\psi$ are the standard deviation of output values of $\varsigma$, the noise standard deviation and a constant, respectively. The determination of $\sigma$ and $\psi$ is explained in section 4.

For the solution of this problem it is more appropriate to solve the so-called dual problem [8], which is derived applying the Karush-Kuhn-Tuker (KKT) [8] conditions to (6). The dual formulation is as follows:

$$
\underset{\alpha}{\operatorname{Max}}-\frac{1}{2} \sum_{\mathrm{i}=1 \mathrm{j}=1}^{\mathrm{N}} \sum_{\mathrm{N}}^{\mathrm{N}}\left(\alpha_{\mathrm{i}}-\alpha_{\mathrm{i}}^{*}\right)\left(\alpha_{\mathrm{j}}-\alpha_{\mathrm{j}}^{*}\right) \mathrm{K}\left(\mathrm{x}_{\mathrm{i}}, \mathrm{x}_{\mathrm{j}}\right)-\varepsilon \sum_{\mathrm{i}=1}^{\mathrm{N}}\left(\alpha_{\mathrm{i}}+\alpha_{\mathrm{i}}^{*}\right)+\sum_{\mathrm{i}=1}^{\mathrm{N}} \mathrm{y}_{\mathrm{i}}\left(\alpha_{\mathrm{i}}-\alpha_{\mathrm{i}}^{*}\right)
$$

subject to :

$$
\begin{aligned}
& \sum_{i=1}^{N}\left(\alpha_{i}-\alpha_{i}^{*}\right)=0 \\
& \alpha_{i}, \alpha_{i}^{*} \in[0, C]
\end{aligned}
$$

The solution of (7) is given by $\boldsymbol{\alpha}$, which is a vector of Lagrange Multipliers, where the values that satisfy $\left(\alpha_{i}-\alpha_{i}^{*}\right) \neq 0$ correspond to the sv of the problem. Once sv are determined, the regression function $f$ is given by:

$$
f(\mathbf{x})=\sum_{i=1}^{N}\left(\alpha_{i}-\alpha_{i}^{*}\right) K\left(\mathbf{x}, \mathbf{x}_{i}\right)+b
$$

This function is non-linear in the input space, but linear in a high dimensional feature space. The translation of input data to a higher dimensional space is possible because the use of $K(\bullet)$ in (7). These functions must satisfy certain known conditions to be admissible as kernels in a SVM. Specifically they must satisfy Mercer's condition [6]. Many functions may be used as kernels [10], but the most popular are: a) Polynomial learning machines (PLM), b) Radial-basis functions (RBF) and c) Twolayer perceptrons (LP) [6].

In this paper, the selection of Polynomial learning results very appropriate for the construction of an explicit algebraic expression, as we explain below.

\subsection{Polynomial Kernel}

As shown in (8), $f(\boldsymbol{x})$ is determined by the kernel function and the values of $\boldsymbol{x}$ that appear in that expression as function of $K(.,$.$) . The theory of SVM asserts the con-$ struction of Kernels of the form $K\left(\mathbf{x}, \mathbf{x}_{i}\right)=\varphi(\mathbf{x})^{T} \varphi\left(\mathbf{x}_{i}\right) ; \varphi($.$) is a mapping that allows$ the transformation of points from input space to higher dimensional spaces. The knowledge of $\varphi($.) is relevant because it allows $f(x)$ to be expressed in an explicit way and shows the functional relation involved between independent variables of some specific problem. 
However, for many kernels the functional $\varphi($.$) is given implicitly and it is very dif-$ ficult (even impossible) to derive it form the particular Kernel chosen. For example, if a Radial Basis Function Kernel is used, it is very difficult to propose a function $\varphi($.$) , such that \varphi(\mathbf{x})^{T} . \varphi\left(\mathbf{x}_{i}\right)=\tanh \left(\beta_{0} \mathbf{x}^{T} \mathbf{x}_{i}+\beta_{1}\right)$.

In the case of polynomial kernel, $\varphi($.$) can be known because it represents a poly-$ nomial expression. Its monomials are combinations of independent variables with distinct exponents. The polynomial kernel is given by $K\left(\mathbf{x}, \mathbf{x}_{i}\right)=\left(\beta_{0} \mathbf{x}^{T} \mathbf{x}_{i}+\beta_{1}\right)^{\rho}$. In this paper, we consider $\beta_{0}=\beta_{1}=1$ and applying recursively the Newton's binomial theorem, this Kernel can be expanded as:

$$
\begin{aligned}
& \left(1+\mathbf{x}^{T} \mathbf{x}_{i}\right)^{\rho}=\left(1+\sum_{j=1}^{k} x_{j} x_{i j}\right)^{\rho} \\
& =\sum_{i=1}^{\rho}\left(\begin{array}{l}
\rho \\
i_{1}
\end{array}\right)\left[\sum_{j=1}^{k} x_{j} x_{j i}\right]^{\rho-i_{1}} \\
& =\sum_{i_{1}=1}^{\rho}\left(\begin{array}{l}
\rho \\
i_{1}
\end{array}\right) \sum_{i_{2}=1}^{\rho-i_{1}}\left(\begin{array}{c}
\rho-i_{1} \\
i_{2}
\end{array}\right)\left(x_{1} x_{i 1}\right)^{i_{2}}\left[\sum_{j=2}^{k} x_{j} x_{i j}\right]^{\rho-i_{1}-i_{2}} \\
& =\sum_{i_{1}=1}^{\rho}\left(\begin{array}{l}
\rho \\
i_{1}
\end{array}\right) \sum_{i_{2}=1}^{\rho-i_{1}}\left(\begin{array}{c}
\rho-i_{1} \\
i_{2}
\end{array}\right)\left(x_{1} x_{i 1}\right)^{i_{2}} \cdots \sum_{i_{k}=1}^{\rho-\sum_{j=1}^{k-1} i_{j}}\left(\begin{array}{c}
\rho-\sum_{j=1}^{k-1} i_{j} \\
i_{2}
\end{array}\right)\left(x_{k-1} x_{i k-1}\right)^{i_{k}}\left(x_{k} x_{i k}\right)^{\rho-\sum_{j=1}^{k} i_{j}}
\end{aligned}
$$

With (9) we conclude that the binomial coefficients for a polynomial with degree $\rho$ and $k$ independent variables can be obtained from the following expression:

$$
\left(\begin{array}{c}
\rho \\
\mathrm{i}_{1}
\end{array}\right)\left(\begin{array}{c}
\rho-i_{1} \\
\mathrm{i}_{2}
\end{array}\right)\left(\begin{array}{c}
\rho-\mathrm{i}_{1}-\mathrm{i}_{2} \\
\mathrm{i}_{3}
\end{array}\right) \ldots\left(\begin{array}{c}
\rho-\sum_{j=1}^{k-1} i_{j} \\
\mathrm{i}_{\mathrm{k}}
\end{array}\right)
$$

The number of elements in the vector $\varphi($.$) is equal to the number n_{m}$ of monomials in (9), where $\varphi\left(\mathbf{x}_{i}\right)$ contains the section of the corresponding monomial where terms $\boldsymbol{x}_{i}$ appears $\left(x_{\mathrm{i} 1}, x_{\mathrm{i} 2}, \ldots, x_{\mathrm{ik}}\right)$ and $\varphi(\mathbf{x})$ contains the section of the monomial where elements of $\boldsymbol{x}$ appears $\left(x_{1}, x_{2}, \ldots, x_{k}\right)$. In each case, both terms are multiplied by the square root of the coefficient monomial's given by (10). For instance, if $\rho=2$ and $k=2, \varphi\left(\mathbf{x}_{i}\right), \varphi(\mathbf{x})$ and $K\left(\boldsymbol{x}, \boldsymbol{x}_{i}\right)$ we have:

$$
\begin{aligned}
& \varphi(\mathbf{x})^{T}=\left(x_{1}^{2}, \sqrt{2} x_{1} x_{2}, x_{2}^{2}, \sqrt{2} x_{1}, \sqrt{2} x_{2}, 1\right) \\
& \varphi\left(\mathbf{x}_{i}\right)^{T}=\left(x_{i 1}^{2}, \sqrt{2} x_{i 1} x_{i 2}, x_{i 2}^{2}, \sqrt{2} x_{i 1}, \sqrt{2} x_{i 2}, 1\right) \\
& K\left(\mathbf{x}, \mathbf{x}_{i}\right)=\varphi(\mathbf{x})^{T} . \varphi\left(\mathbf{x}_{i}\right)=x_{1}^{2} x_{i 1}^{2}+2 x_{1} x_{2} x_{i 1} x_{i 2}+x_{2}^{2} x_{i 2}^{2}+2 x_{1} x_{i 1}+2 x_{2} x_{i 2}+1
\end{aligned}
$$

Finally, $f(x)$ can be obtained from (8) and (9) once the sv are estimated. The coefficients of $f(\boldsymbol{x})$ are combinations of $\left(\alpha_{i}-\alpha_{i}^{*}\right) \neq 0$ and binomial coefficients (10). As in 
the case of GMPs, the $C_{i_{1} i_{2} \ldots i_{m}}$ are the coefficients of the final expression, where $i_{j}$ is the power of the $j$-th independent variable for that particular monomial.

\section{Experiments}

A set of two nonlinear regression problems is presented here in order to illustrate the application of GMPs and SVMs methodologies. The set of problems are explained as follows:

mpg. This data is available in UCI Machine Learning Repository ${ }^{2}$. The data concerns city-cycle fuel consumption in miles per gallon, to be predicted in terms of 3 multivalued discrete and 4 continuous attributes: 1) cylinders, 2) model year, 3) origin, 4) displacement, 5) horse power, 6) weight and 7) acceleration. Attributes 1 and 2 were eliminated from our analysis because they turned out to be highly correlated. We considered 392 instances in this dataset.

bodyfat. This dataset was obtained from the StatLib--Datasets Archive ${ }^{3}$ and corresponds to some estimates of the percentage of body fat determined by underwater weighing and various body circumference measurements for $252 \mathrm{men}$. The dataset consists of 14 independent variables: 1) Percent body fat, 2) Age (years), 3) Weight (lbs), 4) Height (inches), 5) Neck circumference (cm), 6) Chest circumference $(\mathrm{cm})$, 7) Abdomen 2 circumference $(\mathrm{cm}), 8)$ Hip circumference $(\mathrm{cm}), 9)$ Thigh circumference $(\mathrm{cm}), 10)$ Knee circumference $(\mathrm{cm}), 11)$ Ankle circumference $(\mathrm{cm}), 12)$ Biceps (extended) circumference $(\mathrm{cm}), 13)$ Forearm circumference $(\mathrm{cm}), 14)$ Wrist circumference $(\mathrm{cm})$.

\subsection{Results of GMPs and SVMs Applied in mpg}

The experiments we performed for in this article (applying GMPs and SVMs to the mpg dataset) are:

GMPs. The VGA was run in this experiment with probability of crossover equal to $0.9(\mathrm{Pc}=0.9)$ and probability of mutation of $0.005(\mathrm{Pm}=0.005)^{4}$. The number of individuals was 50 (np) over 50 (ng) generations. The maximum value allowed for di was 2 , for the 5 independent variables. The number of monomials was $\gamma=20$. The RMS of this method was $14.5 \%$. The coefficients are shown in Table 1.

SVMs. A Polynomial Kernel of degree $\rho=3^{5}$ was applied here. The value of $C=0.62$ was determined as explained in section 3 . The noise variance $\sigma^{2}$ was calculated with the equation $\hat{\sigma}^{2}=\frac{1}{n-d} \sum_{i=1}^{N}\left(y_{i}-\hat{y}_{i}\right)^{2} \quad[11]$, where $\hat{y}_{i}$ is the output of a polynomial

\footnotetext{
${ }^{2}$ http://www.ics.uci.edu/ mlearn/MLSummary.html.

${ }^{3}$ http://lib.stat.cmu.edu/datasets/.

${ }^{4}$ The values for $\mathrm{P}_{\mathrm{c}}$ and $\mathrm{P}_{\mathrm{m}}$ were chosen after vast experimentation with several plausible values.

${ }^{5}$ A value of $\rho=3$ allows more coefficients of Polynomial SVM to match with different coefficients of GMPs (given $d_{i}=2$ ).
} 
SVM with degree $d=5$ (degrees of freedom). The value of $\psi$ was set to 3 , as is done in [11]. Once $\sigma$ and $\psi$ are determined, the value of $\varepsilon$ used for training was 0.0228 . With a polynomial Kernel of degree 3 and 5 independent variables, the total number of monomials that represent $f(x)$ is 56 . All of them are shown in table 1. The RMS of this method was 6.6\%. We use LIBSVM (ver 2.81) in our experiments [12].

Table 1. Results of GMPs and SVMs methods for mpg problem

\begin{tabular}{|c|c|c|c|c|c|c|c|c|}
\hline COEF & SVM & GMP & COEF & SVM & GMP & COEF & SVM & GMP \\
\hline $\mathrm{C} 00003$ & 0.02 & - & $\mathrm{C} 10101$ & -0.09 & - & $\mathrm{C} 10010$ & -0.16 & - \\
\hline $\mathrm{C} 00012$ & -0.04 & - & $\mathrm{C} 10110$ & -0.17 & - & $\mathrm{C} 10100$ & 0.02 & - \\
\hline $\mathrm{C} 00021$ & 0.15 & - & $\mathrm{C} 10200$ & 0.07 & - & $\mathrm{C} 11000$ & 0.02 & - \\
\hline $\mathrm{C} 00030$ & -0.21 & - & $\mathrm{C} 11001$ & -0.14 & - & $\mathrm{C} 20000$ & -0.06 & - \\
\hline $\mathrm{C} 00102$ & -0.09 & - & $\mathrm{C} 11010$ & 0.14 & - & $\mathrm{C} 00001$ & -0.02 & - \\
\hline $\mathrm{C} 00111$ & -0.05 & - & $\mathrm{C} 11100$ & -0.02 & - & $\mathrm{C} 00010$ & 0.10 & - \\
\hline $\mathrm{C} 00120$ & -0.03 & - & $\mathrm{C} 12000$ & 0.05 & - & $\mathrm{C} 00100$ & 0.00 & - \\
\hline $\mathrm{C} 00201$ & 0.11 & - & $\mathrm{C} 20001$ & -0.04 & - & $\mathrm{C} 01000$ & -0.33 & - \\
\hline $\mathrm{C} 00210$ & 0.24 & 0.57 & $\mathrm{C} 20010$ & 0.09 & - & $\mathrm{C} 10000$ & -0.10 & -0.70 \\
\hline $\mathrm{C} 00300$ & -0.08 & - & $\mathrm{C} 20100$ & -0.14 & - & $\mathrm{C} 00000$ & 0.57 & 0.72 \\
\hline $\mathrm{C} 01002$ & -0.02 & - & $\mathrm{C} 21000$ & 0.06 & - & $\mathrm{C} 02020$ & - & -2.35 \\
\hline $\mathrm{C} 01011$ & 0.26 & - & $\mathrm{C} 30000$ & 0.03 & - & $\mathrm{C} 01112$ & - & 3.84 \\
\hline $\mathrm{C} 01020$ & 0.00 & 1.81 & $\mathrm{C} 00002$ & 0.01 & - & $\mathrm{C} 11222$ & - & 0.88 \\
\hline $\mathrm{C} 01101$ & -0.10 & - & $\mathrm{C} 00011$ & -0.11 & - & $\mathrm{C} 12002$ & - & 2.35 \\
\hline $\mathrm{C} 01110$ & -0.01 & - & $\mathrm{C} 00020$ & 0.23 & - & $\mathrm{C} 12100$ & - & 5.48 \\
\hline $\mathrm{C} 01200$ & 0.03 & - & $\mathrm{C} 00101$ & 0.12 & - & $\mathrm{C} 10221$ & - & -0.92 \\
\hline $\mathrm{C} 02001$ & -0.09 & 2.33 & $\mathrm{C} 00110$ & -0.02 & -0.30 & $\mathrm{C} 11102$ & - & -13.94 \\
\hline $\mathrm{C} 02010$ & 0.17 & - & $\mathrm{C} 00200$ & -0.12 & - & $\mathrm{C} 11122$ & - & -14.40 \\
\hline $\mathrm{C} 02100$ & 0.15 & - & $\mathrm{C} 01001$ & 0.04 & - & $\mathrm{C} 12201$ & - & 15.99 \\
\hline $\mathrm{C} 03000$ & 0.02 & - & $\mathrm{C} 01010$ & -0.20 & - & $\mathrm{C} 21211$ & - & 165.70 \\
\hline $\mathrm{C} 10002$ & 0.06 & - & $\mathrm{C} 01100$ & -0.07 & -2.05 & $\mathrm{C} 22100$ & - & -3.00 \\
\hline $\mathrm{C} 10011$ & -0.08 & - & $\mathrm{C} 02000$ & 0.07 & -0.04 & $\mathrm{C} 22211$ & - & -425.66 \\
\hline $\mathrm{C} 10020$ & 0.07 & - & $\mathrm{C} 10001$ & 0.04 & - & & & \\
\hline
\end{tabular}

\subsection{Results of GMPs and SVMs Applied in Bodyfat}

The experiments applied to bodyfat dataset with GMPs and SVMs are as follows:

GMPs. The parameters used for VGA in this experiment were: $P_{c}=1.0, P_{m}=0.005^{4}$, $n_{p}=50$ and $n_{p}=50$. The maximum value allow for $d_{i}$ was 1 , for the 14 independent variables. The number of monomials was $\gamma=20$. The RMS of this method was $8.0 \%$. The coefficients are shown in Table 2.

SVMs. A Polynomial Kernel of degree $\rho=2$ was applied here. The value of $C$ was 0.5 and the value of $\varepsilon=0.024$, where the noise variance was also estimated applying first a 
polynomial SVM with $d=5$ degrees of freedom. With a polynomial kernel of degree 2 and 14 independent variables, the total number of monomials that represent $f(x)$ is 120. Only those coefficients that also appear in the GMP approach are shown in Table 2 , the rest (118) are not displayed because of lack of space. The RMS for this problem was $2.5 \%$. Likewise, we used LIBSVM (ver 2.81) [12].

Table 2. Results of GMPs and SVMs methods for bodyfat problem

\begin{tabular}{|l|c|c|c|c|c|}
\hline \multicolumn{1}{|c|}{ COEF } & SVM & GMP & COEF & SVM & GMP \\
\hline C01001000000000 & 0.005 & -1.27 & C10010100110000 & - & 8.740 \\
\hline C00000000000000 & 0.871 & 0.93 & C11000111000100 & - & 7.475 \\
\hline C00010110111000 & - & -15.81 & C10011001100010 & - & -2.069 \\
\hline C00011001010111 & - & -22.50 & $\mathrm{C} 01010101100010$ & - & -20.024 \\
\hline C00100111111001 & - & 13.37 & $\mathrm{C} 10100000001110$ & - & 1.657 \\
\hline C00001110111101 & - & 53.13 & $\mathrm{C} 11000110011011$ & - & -116.780 \\
\hline $\mathrm{C} 00100000101111$ & - & -6.17 & $\mathrm{C} 10110110001011$ & - & 43.529 \\
\hline $\mathrm{C} 01100010110100$ & - & -16.57 & $\mathrm{C} 11001101011011$ & - & 78.219 \\
\hline $\mathrm{C} 01011011101101$ & - & 26.18 & $\mathrm{C} 11100100100111$ & - & -34.857 \\
\hline $\mathrm{C} 01011110100011$ & - & 34.23 & $\mathrm{C} 01001000110010$ & - & 6.291 \\
\hline
\end{tabular}

\section{Conclusions}

From both of our methods we are able to achieve the explicit algebraic representation of the behavior of complex dynamic systems. This is a goal that has eluded many researchers in the past. We believe that this is a major contribution of our paper. In determining which approach is better we have to point out that SVMs are theoretically sounder than GMPs. However, as shown in the previous experiments, the number of monomials in SVM approach is substantially greater than those obtained with GMPs. Even though the accuracy of SVMs is superior in both experiments, this is so only at the expense of having to consider larger sets of monomials. The results also suggest that GMP can approximate very well, displaying an explicit algebraic expression with a relatively few number of terms (monomials). Many more experiments are necessary to establish a proper comparison between these methods. In this paper we focused on the fact that GMPs and SVMs with polynomial kernels can build similar expressions for some tasks, particularly nonlinear regression problems. A more detailed analysis with other kind of problems is a matter for future work.

\section{References}

1. Salcedo, S., Fernández, J., Segovia, M., Bousoño, C.: Genetic programming for the prediction of insolvency in non-life insurance companies, Computers \& Operations Research 32 (2005) 749-765.

2. Kuri, A., Approximation and Classification with Genetic Multivariate Polynomials, WSEAS Transactions on Computers, Issue 3, (2006) 645-652. 
3. Rudolph, G.: Convergence Analysis of Canonical Genetic Algorithms, IEEE Transactions on Neural Networks, Vol. 5, Issue 1, (1994) 96-101.

4. Kuri, A.: A Methodology for the Statistical Characterization of Genetic Algorithms. Proceedings of the Mexican International Congress on Artificial Intelligence, SpringerVerlag, (2002) 79-88.

5. Boser, E., Guyon, I., Vapnik, V.: A training algorithm for optimal margin classifiers. 5th Annual ACM Workshop on COLT, ACM Press, (1992) 144-152.

6. Haykin, S.: Neural Networks. A comprehensive foundation. 2nd ed., Prentice Hall, New Jersey (1999).

7. Chapelle, O., Haffner, P., Vapnik, V.: Support vector for histogram-based image classification. IEEE transactions on Neural Networks Vol. 10, 5, (1999) 1055-1065.

8. Smola, A., Schölkopf B.: A Tutorial on Support Vector Regression. NeuroCOLT Technical Report NC-TR-98-030, Royal Holloway College, University of London, UK, (1998).

9. Kuri, A., Mejía, I.: Evolutionary Training of SVM for Classification Problems with SelfAdaptive Parameters. Advances in Artificial Intelligence Theory, (Gelbukh, A., Monroy, R., Eds.), IPN, (2005) 207-216.

10. Vapnik, V.N.: The Nature of Statistical Learning Theory. Springer-Verlag, NY, (1995).

11. Cherkassky, V., Ma, Y.: Practical Selection of SVM Parameters and Noise Estimation for SVM Regression. Neural Networks, Vol. 17 No. 1, (2004) 113-126.

12. Chang, C., Lin, C.: LIBSVM: a library for support vector machines, (2001). Software available at http://www.csie.ntu.edu.tw/ cjlin/libsvm/.

13. Choi, M. D.: Tricks or Treats with the Hilbert Matrix. American Mathematical Monthly Vol. 90, (1983) 301-312. 